\title{
Sharing three values with small weights
}

\author{
Indrajit LAHIRI
}

(Received September 2, 2005)

\begin{abstract}
We prove a uniqueness theorem for meromorphic functions sharing three values with small weights which improves some known results. We also exhibit some applications of the main result.
\end{abstract}

Key words: weighted sharing, uniqueness, meromorphic functions.

\section{Introduction, Definitions and Results}

Let $f$ and $g$ be two nonconstant meromorphic functions defined in the open complex plane $\mathbb{C}$. For $b \in \mathbb{C} \cup\{\infty\}$ we say that $f$ and $g$ share the value $b \mathrm{CM}$ (counting multiplicities) if $f$ and $g$ have the same $b$-points with the same multiplicities. If we do not take multiplicities into account, we say that $f$ and $g$ share the value $b$ IM (ignoring multiplicities). For standard definitions and notations of the value distribution theory we refer [1].

H. Ueda [9] proved the following result

Theorem A ([9]) Let $f$ and $g$ be two distinct nonconstant entire functions sharing $0,1 C M$ and let $a(\neq 0,1)$ be a finite complex number. If a is lacunary for $f$ then $1-a$ is lacunary for $g$ and $(f-a)(g+a-1) \equiv a(1-a)$.

Improving Theorem A H.X. Yi [11] proved the following theorem.

Theorem B ([11]) Let $f$ and $g$ be two distinct nonconstant entire functions sharing $0,1 C M$ and let $a(\neq 0,1)$ be a finite complex number. If $\delta(a ; f)>1 / 3$ then $a$ and $1-a$ are Picard exceptional values of $f$ and $g$ respectively and $(f-a)(g+a-1) \equiv a(1-a)$.

Extending Theorem $B$ to meromorphic functions S.Z. Ye [10] proved the following results.

Theorem C ([10]) Let $f$ and $g$ be two distinct nonconstant meromorphic functions such that $f$ and $g$ share $0,1, \infty C M$. Let $a(\neq 0,1)$ be a finite complex number. If $\delta(a ; f)+\delta(\infty ; f)>4 / 3$ then a and $1-a$ are Picard

2000 Mathematics Subject Classification : 30D35. 
exceptional values of $f$ and $g$ respectively and also $\infty$ is so and $(f-a)(g+$ $a-1) \equiv a(1-a)$.

Theorem D ([10]) Let $f$ and $g$ be two distinct nonconstant meromorphic functions sharing $0,1, \infty C M$. Let $a_{1}, a_{2}, \ldots, a_{p}$ be $p(\geq 1)$ distinct finite complex numbers and $a_{j} \neq 0,1$ for $j=1,2,3, \ldots, p$. If $\sum_{j=1}^{p} \delta\left(a_{j} ; f\right)+$ $\delta(\infty ; f)>2(p+1) /(p+2)$ then there exist one and only one $a_{k}$ in $a_{1}, a_{2}, \ldots$, $a_{p}$ such that $a_{k}$ and $1-a_{k}$ are Picard exceptional values of $f$ and $g$ respectively and also $\infty$ is so and $\left(f-a_{k}\right)\left(g+a_{k}-1\right) \equiv a_{k}\left(1-a_{k}\right)$.

Improving above results H.X. Yi [12] proved the following theorem.

Theorem E ([12]) Let $f$ and $g$ be two distinct nonconstant meromorphic functions such that $f$ and $g$ share $0,1, \infty C M$. Let $a(\neq 0,1)$ be a finite complex number. If $N(r, a ; f) \neq T(r, f)+S(r, f)$ and $N(r, f) \neq T(r, f)+$ $S(r, f)$ then a and $1-a$ are Picard exceptional values of $f$ and $g$ respectively and also $\infty$ is so and $(f-a)(g+a-1) \equiv a(1-a)$.

Definition 1 Let $p$ be a positive integer and $b \in \mathbb{C} \cup\{\infty\}$. Then by $N(r, b ; f \mid \leq p)$ we denote the counting function of those $b$-points of $f$ (counted with proper multiplicities) whose multiplicities are not greater than $p$. By $\bar{N}(r, b ; f \mid \leq p)$ we denote the corresponding reduced counting function.

In an analogous manner we define $N(r, b ; f \mid \geq p)$ and $\bar{N}(r, b ; f \mid \geq p)$.

Also we put

$$
\delta_{p)}(a ; f)=1-\limsup _{r \rightarrow \infty} \frac{N(r, a ; f \mid \leq p)}{T(r, f)} .
$$

Hua and Fang [2] proved that if two nonconstant distinct meromorphic functions $f$ and $g$ share $0,1, \infty \mathrm{CM}$ then $N(r, a ; f \mid \geq 3)=S(r, f)$ for any complex number $a(\neq 0,1, \infty)$.

Also Yi [12] proved that if two nonconstant distinct meromorphic functions $f$ and $g$ share $0,1, \infty$ CM then $N(r, \infty ; f \mid \geq 2)=S(r, f)$.

Therefore Theorem $E$ of $\mathrm{Yi}$ can easily be improved to the following result.

Theorem F ([5]) Let $f$ and $g$ be distinct nonconstant meromorphic functions sharing $0,1, \infty$ CM. If a $(\neq 0,1)$ is a finite complex number such that $N(r, a ; f \mid \leq 2) \neq T(r, f)+S(r, f)$ and $N(r, \infty ; f \mid \leq 1) \neq T(r, f)+S(r, f)$ then $a$ and $1-a$ are Picard exceptional values of $f$ and $g$ respectively and 
also $\infty$ is so and $(f-a)(g+a-1) \equiv a(1-a)$.

Following examples show that Theorem $F$ is sharp.

Example 1 ([5]) Let $f=\left(e^{z}-1\right) /\left(e^{z}+1\right), g=\left(1-e^{z}\right) /\left(1+e^{z}\right), a_{1}=$ -1 and $a_{2}=2$. Then $f, g$ share $0,1, \infty$ CM. Also $N(r, \infty ; f \mid \leq 1)=$ $T(r, f)+S(r, f), N\left(r, a_{1} ; f \mid \leq 2\right) \neq T(r, f)+S(r, f)$ and $N\left(r, a_{2} ; f \mid \leq\right.$ $2)=T(r, f)+S(r, f)$. Clearly $\left(f-a_{i}\right)\left(g+a_{i}-1\right) \not \equiv a_{i}\left(1-a_{i}\right)$ for $i=1,2$.

Example 2 ([5]) Let $f=e^{z}, g=e^{-z}$ and $a=2$. Then $f, g$ share $0,1, \infty$ CM. Also $N(r, \infty ; f \mid \leq 1) \neq T(r, f)+S(r, f), N(r, a ; f \mid \leq 2)=$ $T(r, f)+S(r, f)$. Clearly $(f-a)(g+a-1) \not \equiv a(1-a)$.

It is shown in [5] by the following example that the condition $N(r, a ; f \mid$ $\leq 2) \neq T(r, f)+S(r, f)$ of Theorem $F$ cannot be replaced by any one of $N(r, a ; f \mid \leq 1) \neq T(r, f)+S(r, f)$ and $\bar{N}(r, a ; f \mid \leq 2) \neq T(r, f)+S(r, f)$.

Example 3 ([5]) Let $f=e^{z}\left(1-e^{z}\right), g=e^{-z}\left(1-e^{-z}\right)$ and $a=1 / 4$. Then $f, g$ share $0,1, \infty$ CM. Also $N(r, \infty ; f \mid \leq 1) \neq T(r, f)+S(r, f)$. Since $f-a=-\left(e^{z}-2 a\right)^{2}$, we see the following

(i) $N(r, a ; f \mid \leq 1) \equiv 0$,

(ii) $\bar{N}(r, a ; f \mid \leq 2)=N\left(r, 2 a ; e^{z}\right)=(1 / 2) T(r, f)+S(r, f)$ and

(iii) $N(r, a ; f \mid \leq 2)=2 N\left(r, 2 a ; e^{z}\right)=T(r, f)+S(r, f)$.

Also clearly $(f-a)(g+a-1) \not \equiv a(1-a)$.

Following two examples show that in the above theorems the sharing of 0 and 1 can not be relaxed from CM to IM.

Example 4 ([5]) Let $f=e^{z}-1, g=\left(e^{z}-1\right)^{2}$ and $a=-1$. Then $f$, $g$ share $0 \mathrm{IM}$ and $1, \infty \mathrm{CM}$. Also $N(r, \infty ; f) \equiv 0$ and $N(r, a ; f) \equiv 0$ but $(f-a)(g+a-1) \not \equiv a(1-a)$.

Example 5 ([5]) Let $f=2-e^{z}, g=e^{z}\left(2-e^{z}\right)$ and $a=2$. Then $f$, $g$ share $1 \mathrm{IM}$ and $0, \infty \mathrm{CM}$. Also $N(r, \infty ; f) \equiv 0$ and $N(r, a ; f) \equiv 0$ but $(f-a)(g+a-1) \not \equiv a(1-a)$.

In [5] following question is asked: Is it really impossible to relax in any way the nature of sharing of any one of 0 and 1 in the above theorems?

The notion of weighted sharing of values is used in [5] to deal this problem. We now explain the notion in the following definition which measures how close a shared value is to being shared CM or to being shared IM. 
Definition $2([3,4])$ Let $k$ be a nonnegative integer or infinity. For $a \in$ $\mathbb{C} \cup\{\infty\}$ we denote by $E_{k}(a ; f)$ the set of all $a$-points of $\mathrm{f}$ where an $a$-point of multiplicity $m$ is counted $m$ times if $m \leq k$ and $k+1$ times if $m>k$. If $E_{k}(a ; f)=E_{k}(a ; g)$, we say that $f, g$ share the value $a$ with weight $k$.

The definition implies that if $f, g$ share a value $a$ with weight $k$ then $z_{o}$ is a zero of $f-a$ with multiplicity $m(\leq k)$ if and only if it is a zero of $g-a$ with multiplicity $m(\leq k)$ and $z_{o}$ is a zero of $f-a$ with multiplicity $m(>k)$ if and only if it is a zero of $g-a$ with multiplicity $n(>k)$ where $m$ is not necessarily equal to $n$.

We write $f, g$ share $(a, k)$ to mean that $f, g$ share the value $a$ with weight $k$. Clearly if $f, g$ share $(a, k)$ then $f, g$ share $(a, p)$ for all integer $p$, $0 \leq p<k$. Also we note that $f, g$ share a value $a$ IM or CM if and only if $f, g$ share $(a, 0)$ or $(a, \infty)$ respectively.

Improving Theorem $C$ in [5] following result is proved.

Theorem G ([5]) Let $f$ and $g$ be two distinct meromorphic functions sharing $(0,1),(1, \infty)$ and $(\infty, \infty)$. If $a(\neq 0,1)$ is a finite complex number such that $3 \delta_{2)}(a ; f)+2 \delta_{1)}(\infty ; f)>3$ then $a$ and $1-a$ are Picard exceptional values of $f$ and $g$ and also $\infty$ is so and $(f-a)(g+a-1) \equiv a(1-a)$.

In [5] we were unable to relax the nature of sharing of values in Theorem $F$. We now take up this problem and prove the following result which improve Theorem $F$ and so all previous results.

Theorem 1 Let $f$ and $g$ be two distinct meromorphic functions sharing $(0,1),(1, m)$ and $(\infty, k)$, where $(m-1)(m k-1)>(1+m)^{2}$. If $a(\neq 0,1)$ is a finite complex number such that $N(r, a ; f \mid \leq 2) \neq T(r, f)+S(r, f)$ and $N(r, \infty ; f \mid \leq 1) \neq T(r, f)+S(r, f)$ then $a$ and $1-a$ are Picard exceptional values of $f$ and $g$ respectively and also $\infty$ is so and $(f-a)(g+a-1) \equiv$ $a(1-a)$.

We note that the condition $(m-1)(m k-1)>(1+m)^{2}$ is equivalent to $(m-1)(k-1)>4$ and so is symmetric in $m$ and $k$. We also note that Theorem 1 holds for the following pairs of least values of $m$ and $k$ : (i) $m=$ $3, k=4$; (ii) $m=4, k=3$; (iii) $m=2, k=6$; (iv) $m=6, k=2$.

Definition 3 Let $f$ and $g$ share a value $a$ IM. Let $z$ be an $a$-point of $f$ and $g$ with multiplicities $p_{f}(z)$ and $p_{g}(z)$ respectively.

We put 


$$
\begin{array}{rlrl}
\bar{\nu}_{f}(z)=1 & \text { if } p_{f}(z)>p_{g}(z) \\
& =0 & & \text { if } p_{f}(z) \leq p_{g}(z)
\end{array}
$$

and

$$
\begin{aligned}
\bar{\mu}_{f}(z)=1 & \text { if } p_{f}(z)<p_{g}(z) \\
=0 & \text { if } p_{f}(z) \geq p_{g}(z) .
\end{aligned}
$$

Let $\bar{n}(r, a ; f>g)=\sum_{|z| \leq r} \bar{\nu}_{f}(z)$ and $\bar{n}(r, a ; f<g)=\sum_{|z| \leq r} \bar{\mu}_{f}(z)$. We now denote by $\bar{N}(r, a ; f>g)$ and $\bar{N}(r, a ; f<g)$ the integrated counting functions obtained from $\bar{n}(r, a ; f>g)$ and $\bar{n}(r, a ; f<g)$ respectively.

Finally we put $\bar{N}_{*}(r, a ; f, g)=\bar{N}(r, a ; f>g)+\bar{N}(r, a ; f<g)$.

Definition 4 Let $f$ and $g$ share a value $a$ IM. Let $z$ be an $a$-point of $f$ and $g$ with multiplicities $p_{f}(z)$ and $p_{g}(z)$ respectively.

We put

$$
\begin{aligned}
\nu_{f}(z) & =p_{f}(z) \quad \text { if } p_{f}(z)>p_{g}(z) \\
& =0 \quad \text { if } p_{f}(z) \leq p_{g}(z)
\end{aligned}
$$

and

$$
\begin{aligned}
\mu_{f}(z) & =p_{f}(z) \quad \text { if } p_{f}(z)<p_{g}(z) \\
& =0 \quad \text { if } p_{f}(z) \geq p_{g}(z) .
\end{aligned}
$$

Let $n(r, a ; f>g)=\sum_{|z| \leq r} \nu_{f}(z)$ and $n(r, a ; f<g)=\sum_{|z| \leq r} \mu_{f}(z)$. We now denote by $N(r, a ; f>g)$ and $N(r, a ; f<g)$ the integrated counting functions obtained from $n(r, a ; f>g)$ and $n(r, a ; f<g)$ respectively.

Throughout the paper we denote by $f$ and $g$ two nonconstant meromorphic functions defined in $\mathbb{C}$.

\section{Lemmas}

In this section we present some lemmas which are needed in the sequel.

Lemma 1 ([3]) If $f, g$ share $(0,0),(1,0),(\infty, 0)$ then (i) $T(r, f) \leq$ $3 T(r, g)+S(r, f)$, (ii) $T(r, g) \leq 3 T(r, f)+S(r, g)$.

This shows that $S(r, f)=S(r, g)$ and we denote them by $S(r)$.

Lemma 2 ([6]) Let $f, g$ share $(0,1),(1, m),(\infty, k)$ and $f \not \equiv g$, where $(m-1)(m k-1)>(1+m)^{2}$. Then $\bar{N}(r, a ; f \mid \geq 2)=S(r)$ and $\bar{N}(r, a ; g \mid \geq$ 
2) $=S(r)$ for $a=0,1, \infty$.

Following lemma can be proved in the line of statements (iii) and (iv) of Lemma 2.3 of [7].

Lemma 3 Let $f, g$ share $(0,0),(1,0),(\infty, 0)$ and $f \not \equiv g$. If $\alpha=(f-$ 1) $/(g-1)$ and $h=g / f$ then

(i) $\bar{N}(r, 0 ; \alpha)=\bar{N}(r, \infty ; f<g)+\bar{N}(r, 1 ; f>g)$,

(ii) $\bar{N}(r, \infty ; \alpha)=\bar{N}(r, \infty ; f>g)+\bar{N}(r, 1 ; f<g)$,

(iii) $\bar{N}(r, 0 ; h)=\bar{N}(r, 0 ; f<g)+\bar{N}(r, \infty ; f>g)$,

(iv) $\bar{N}(r, \infty ; h)=\bar{N}(r, 0 ; f>g)+\bar{N}(r, \infty ; f<g)$.

Lemma 4 Let $f, g$ share $(0,1),(1, m),(\infty, k)$ and $f \not \equiv g$, where $(m-$ 1) $(m k-1)>(1+m)^{2}$. If $\alpha$ and $h$ are defined as in Lemma 3 then $\bar{N}(r, a ; \alpha)=S(r)$ and $\bar{N}(r, a ; h)=S(r)$ for $a=0, \infty$.

Proof. The lemma follows from Lemmas 2 and 3 because $\bar{N}_{*}(r, a ; f, g) \leq$ $\bar{N}(r, a ; f \mid \geq 2)$ for $a=0,1, \infty$.

Lemma $5([8]) \quad$ Let $f$ and $g$ share $(0,0),(1,0),(\infty, 0)$. If $f$ is a bilinear transformation of $g$ then $f$ and $g$ satisfy exactly one of the following: ( i ) $f \equiv g$, ( ii ) $f+g \equiv 1$, (iii) $(f-1)(g-1) \equiv 1$, (iv) $f g \equiv 1$, ( v ) $f \equiv$ $A g+1-A$, (vi) $f \equiv A g$, (vii) $f(g+A-1) \equiv A g$, where $A(\neq 0,1)$ is a constant.

Following lemma is of independent interest.

Lemma 6 Let $f, g$ share $(0,1),(1, m),(\infty, k)$ and $f \not \equiv g$, where $(m-$ 1) $(m k-1)>(1+m)^{2}$. If $f$ is not a bilinear transformation of $g$ then each of the following holds:

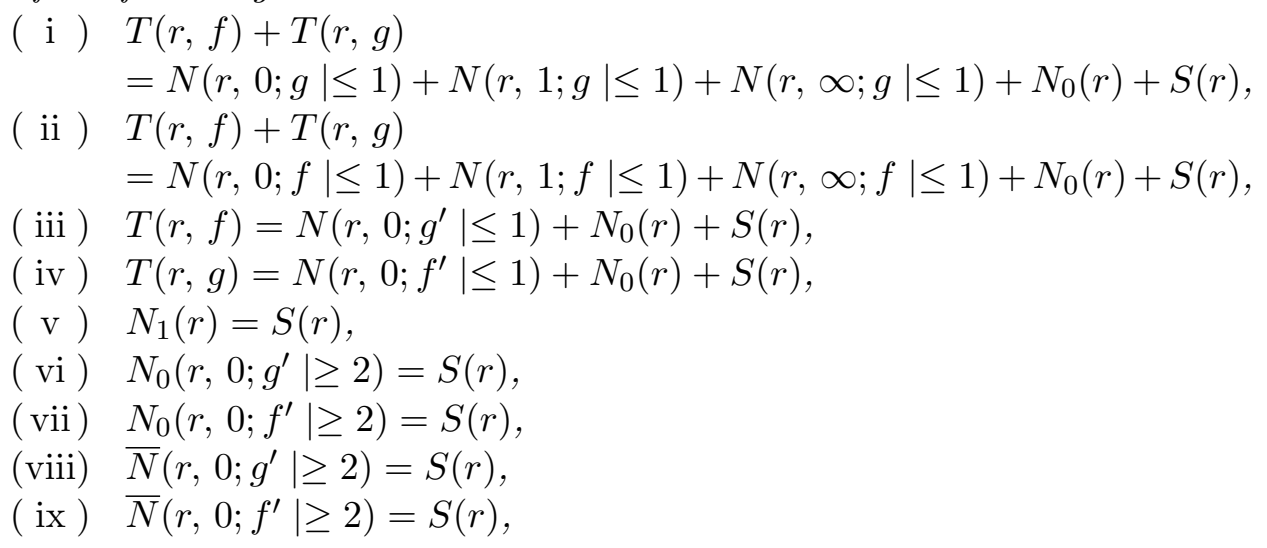


where $N_{0}(r)\left(N_{1}(r)\right)$ denotes the counting function of those simple (multiple) zeros of $f-g$ which are not the zeros of $g(g-1), 1 / g$ and so are not the zeros of $f(f-1), 1 / f$, also $N_{0}\left(r, 0 ; g^{\prime} \mid \geq 2\right)\left(N_{0}\left(r, 0 ; f^{\prime} \mid \geq 2\right)\right)$ is the counting function of those multiple zeros of $g^{\prime}\left(f^{\prime}\right)$ which are not the zeros of $g(g-1)$ and so not of $f(f-1)$.

Proof. We see that $f=(1-\alpha) /(1-\alpha h)$ and $g=(1-\alpha) h /(1-\alpha h)$, where $\alpha$ and $h$ are defined as in Lemma 3. Since $f$ is not a bilinear transformation of $g, \alpha, h$ and $\alpha h$ are nonconstant. Let $b=\alpha^{\prime} h /\left(\alpha h^{\prime}+\alpha^{\prime} h\right)$. Then

$$
f-b=\frac{(1-\alpha)-b(1-\alpha h)}{(1-\alpha h)} .
$$

Let $F=(f-b)(1-\alpha h)=(1-\alpha)-b(1-\alpha h)$. Also $(f-g)(1-\alpha h)=(1-$ $\alpha)(1-h)$ and $(g-1)(1-\alpha h)=h-1$ so that $f-g=(g-1)(\alpha-1)$. Again

$$
\frac{g^{\prime}}{g}=\frac{h^{\prime}(1-\alpha h)+(h-1)\left(\alpha^{\prime} h+\alpha h^{\prime}\right)}{h(1-\alpha)(1-\alpha h)} .
$$

Therefore

$$
\begin{aligned}
\frac{g^{\prime}(g-f)}{g(g-1)} & =\frac{h^{\prime}(1-\alpha h)+(h-1)\left(\alpha^{\prime} h+\alpha h^{\prime}\right)}{h(1-\alpha h)} \\
& =\frac{(1-\alpha)\left(\alpha h^{\prime}+\alpha^{\prime} h\right)-\alpha^{\prime} h(1-\alpha h)}{\alpha h(1-\alpha h)} .
\end{aligned}
$$

Again

$$
\begin{aligned}
(f-b)(1-\alpha h) & =(1-\alpha)-b(1-\alpha h) \\
& =\frac{(1-\alpha)\left(\alpha h^{\prime}+\alpha^{\prime} h\right)-\alpha^{\prime} h(1-\alpha h)}{\alpha h^{\prime}+\alpha^{\prime} h}
\end{aligned}
$$

and so

$$
(f-b) \frac{\alpha h^{\prime}+\alpha^{\prime} h}{\alpha h}=\frac{(1-\alpha)\left(\alpha h^{\prime}+\alpha^{\prime} h\right)-\alpha^{\prime} h(1-\alpha h)}{\alpha h(1-\alpha h)} .
$$

From (1) and (2) we get

$$
\frac{g^{\prime}(g-f)}{g(g-1)}=(f-b)\left(\frac{h^{\prime}}{h}+\frac{\alpha^{\prime}}{\alpha}\right) \text {. }
$$

Since $F^{\prime}=-\alpha^{\prime}-b^{\prime}(1-\alpha h)+b\left(\alpha^{\prime} h+\alpha h^{\prime}\right)=-\alpha^{\prime}-b^{\prime}(1-\alpha h)+\alpha^{\prime} h$, we get

$$
\frac{F^{\prime}}{F}-\frac{\alpha^{\prime}}{\alpha}=\frac{-\alpha^{\prime}-b^{\prime}(1-\alpha h)+\alpha^{\prime} h-\left(\alpha^{\prime} / \alpha\right) F}{F}
$$




$$
\begin{aligned}
& =\frac{(1-\alpha h)\left(-\alpha^{\prime}-b^{\prime} \alpha+b \alpha^{\prime}\right)}{\alpha(f-b)(1-\alpha h)} \\
& =\frac{1}{f-b}\left[\frac{\alpha^{\prime}}{\alpha}(b-1)-b^{\prime}\right]
\end{aligned}
$$

and so

$$
\frac{1}{f-b}=\frac{F^{\prime} / F-\alpha^{\prime} / \alpha}{\left(\alpha^{\prime} / \alpha\right)(b-1)-b^{\prime}} .
$$

Since $T(r, \alpha) \leq T(r, f)+T(r, g)+O(1)$ and $T(r, h) \leq T(r, f)+$ $T(r, g)+O(1)$, in view of Lemmas 1 and 4 we obtain

$$
\begin{aligned}
T\left(r, \frac{\alpha^{\prime}}{\alpha}\right) & =m\left(r, \frac{\alpha^{\prime}}{\alpha}\right)+N\left(r, \frac{\alpha^{\prime}}{\alpha}\right) \\
& \leq \bar{N}(r, 0 ; \alpha)+\bar{N}(r, \infty ; \alpha)+S(r, \alpha)=S(r)
\end{aligned}
$$

and

$$
\begin{aligned}
T\left(r, \frac{h^{\prime}}{h}\right) & =m\left(r, \frac{h^{\prime}}{h}\right)+N\left(r, \frac{h^{\prime}}{h}\right) \\
& \leq \bar{N}(r, 0 ; h)+\bar{N}(r, \infty ; h)+S(r, h)=S(r) .
\end{aligned}
$$

Since $1 / b=1+\alpha h^{\prime} / \alpha^{\prime} h$, we get

$$
\begin{aligned}
T(r, b)=T\left(r, \frac{1}{b}\right)+O(1) & \leq T\left(r, \frac{\alpha}{\alpha^{\prime}}\right)+T\left(r, \frac{h^{\prime}}{h}\right)+O(1) \\
& =T\left(r, \frac{\alpha^{\prime}}{\alpha}\right)+S(r)=S(r) .
\end{aligned}
$$

From (4) we now obtain

$$
m\left(r, \frac{1}{f-b}\right) \leq m\left(r, \frac{F^{\prime}}{F}\right)+S(r)=S(r, f)+S(r)=S(r) .
$$

Since $F^{\prime} / F$ and $\alpha^{\prime} / \alpha$ have no multiple pole and $T\left(r, b^{\prime}\right) \leq 2 T(r, b)+S(r, b)$, it follows from the above and (4) that

$$
\begin{aligned}
N(r, 0 ; f-b \mid \geq 2) & \leq 2 N\left(r, 0 ; \frac{\alpha^{\prime}}{\alpha}(b-1)-b^{\prime}\right)+S(r) \\
& \leq 2 T\left(r, \frac{\alpha^{\prime}}{\alpha}(b-1)-b^{\prime}\right)+S(r) \\
& \leq 2 T\left(r, \frac{\alpha^{\prime}}{\alpha}\right)+2 T(r, b-1)+2 T\left(r, b^{\prime}\right)+S(r) \\
& \leq 2 T\left(r, \frac{\alpha^{\prime}}{\alpha}\right)+6 T(r, b)+S(r)=S(r) .
\end{aligned}
$$


Since $f, g$ share $(0,1),(1, m),(\infty, k)$ and $b=f-(f-b)$, we see that if $z_{0}$ is a zero, pole or 1-point of $g$ which is also a simple zero of $f-b$, then $z_{0}$ is a zero, pole or 1-point of $b$ and so the counting function of such simple zeros of $f-b$ is $S(r)$. So we get from (3) and (6)

$$
\begin{aligned}
N(r, 0 ; f-b) & =N(r, 0 ; f-b \mid \leq 1)+N(r, 0 ; f-b \mid \geq 2) \\
& =N(r, 0 ; f-b \mid \leq 1)+S(r) \\
& =N\left(r, 0 ; g^{\prime} \mid \leq 1\right)+N_{0}(r)+S(r) .
\end{aligned}
$$

From (5) and (7) we obtain

$$
\begin{aligned}
T(r, f) & =T(r, f-b)+S(r) \\
& =m\left(r, \frac{1}{f-b}\right)+N\left(r, \frac{1}{f-b}\right)+S(r) \\
& =N\left(r, 0 ; g^{\prime} \mid \leq 1\right)+N_{0}(r)+S(r),
\end{aligned}
$$

which is (iii).

Similarly we get

$$
T(r, g)=N\left(r, 0 ; f^{\prime} \mid \leq 1\right)+N_{0}(r)+S(r),
$$

which is (iv).

Again from (3) and (6) we obtain $N_{1}(r) \leq N(r, 0 ; f-b \mid \geq 2)+S(r)=$ $S(r)$ and $N_{0}\left(r, 0 ; g^{\prime} \mid \geq 2\right) \leq N(r, 0 ; f-b \mid \geq 2)+S(r)=S(r)$, which are respectively (v) and (vi). Similarly we can prove (vii).

Since $\bar{N}\left(r, 0 ; g^{\prime} \mid \geq 2\right) \leq N_{0}\left(r, 0 ; g^{\prime} \mid \geq 2\right)+\bar{N}(r, 0 ; g \mid \geq 2)+\bar{N}(r, 1 ; g \mid \geq 2)$ and $\bar{N}\left(r, 0 ; f^{\prime} \mid \geq 2\right) \leq N_{0}\left(r, 0 ; f^{\prime} \mid \geq 2\right)+\bar{N}(r, 0 ; f \mid \geq 2)+\bar{N}(r, 1 ; f \mid \geq 2)$, (viii) and (ix) follow from (vi), (vii) and Lemma 2 .

By the second fundamental theorem, Lemma 2 and (8) we get

$$
\begin{aligned}
T(r, f)+ & T(r, g) \\
\leq & T(r, f)+N(r, 0 ; g \mid \leq 1)+N(r, 1 ; g \mid \leq 1) \\
& +N(r, \infty ; g \mid \leq 1)-\bar{N}_{0}\left(r, 0 ; g^{\prime}\right)+S(r) \\
= & N(r, 0 ; g \mid \leq 1)+N(r, 1 ; g \mid \leq 1)+N(r, \infty ; g \mid \leq 1) \\
& +N\left(r, 0 ; g^{\prime} \mid \leq 1\right)+N_{0}(r)-\bar{N}_{0}\left(r, 0 ; g^{\prime}\right)+S(r),
\end{aligned}
$$

where $\bar{N}_{0}\left(r, 0 ; g^{\prime}\right)$ denotes the reduced counting function of those zeros of $g^{\prime}$ which are not the zeros of $g(g-1)$.

By Lemma 2 we see that

$$
N\left(r, 0 ; g^{\prime} \mid \leq 1\right)=N_{0}\left(r, 0 ; g^{\prime} \mid \leq 1\right)+S(r),
$$


where $N_{0}\left(r, 0 ; g^{\prime} \mid \leq 1\right)$ is the counting function of those simple zeros of $g^{\prime}$ which are not the zeros of $g(g-1)$.

Similarly

$$
N\left(r, 0 ; f^{\prime} \mid \leq 1\right)=N_{0}\left(r, 0 ; f^{\prime} \mid \leq 1\right)+S(r) .
$$

From (10) and (11) we get

$$
\begin{aligned}
T(r, f)+ & T(r, g) \\
\leq & N(r, 0 ; g \mid \leq 1)+N(r, 1 ; g \mid \leq 1)+N(r, \infty ; g \mid \leq 1) \\
& +N_{0}\left(r, 0 ; g^{\prime} \mid \leq 1\right)+N_{0}(r)-\bar{N}_{0}\left(r, 0 ; g^{\prime}\right)+S(r) \\
\leq & N(r, 0 ; g \mid \leq 1)+N(r, 1 ; g \mid \leq 1)+N(r, \infty ; g \mid \leq 1) \\
& \quad+N_{0}(r)+S(r) \\
\leq & N(r, 0 ; f-g)+N(r, \infty ; g \mid \leq 1)+S(r) \\
\leq & T(r, f-g)+N(r, \infty ; g \mid \leq 1)+S(r) \\
\leq & m(r, f)+m(r, g) \\
& \quad+N(r, f-g)+N(r, \infty ; g \mid \leq 1)+S(r) \\
\leq & m(r, f)+m(f, g)+N(r, f) \\
& \quad+N(r, \infty ; g>f)+N(r, \infty ; g \mid \leq 1)+S(r) \\
\leq & m(r, f)+N(r, f)+m(r, g)+N(r, g)+S(r) \\
\leq & T(r, f)+T(r, g)+S(r),
\end{aligned}
$$

from which (i) follows.

Now (ii) follows from (i) because $N(r, a ; f \mid \leq 1)=N(r, a ; g \mid \leq 1)$ for $a=0,1, \infty$. This proves the lemma.

Lemma 7 ([6]) Let $f, g$ share $(0,1),(1, m),(\infty, k)$ and $f \not \equiv g$, where $(m-1)(m k-1)>(1+m)^{2}$. Then for any complex number $a(\neq 0,1, \infty)$, $\bar{N}(r, a ; f \mid \geq 3)=S(r)$ and $\bar{N}(r, a ; g \mid \geq 3)=S(r)$.

\section{Proof of the main result}

Proof of Theorem 1. If possible, let $f$ be not a bilinear transformation of $g$. Then by Lemma 6 (vii), Lemma 2, Lemma 7 and the second fundamental theorem we get

$$
\begin{aligned}
2 T(r, f) \leq & N(r, 0 ; f \mid \leq 1)+N(r, 1 ; f \mid \leq 1)+N(r, \infty ; f \mid \leq 1) \\
& +\bar{N}(r, a ; f \mid \leq 2)-N_{1}\left(r, 0 ; f^{\prime} \mid \leq 1\right)+S(r),
\end{aligned}
$$


where $N_{1}\left(r, 0 ; f^{\prime} \mid \leq 1\right)$ is the counting function of those simple zeros of $f^{\prime}$ which are not the zeros of $f(f-1)(f-a)$.

Since a double $a$-point of $f$ is a simple zero of $f^{\prime}$, it follows that

$$
\begin{aligned}
\bar{N}(r, a ; f \mid \leq 2)-N_{1}\left(r, 0 ; f^{\prime} \mid\right. & \leq 1) \\
& =N(r, a ; f \mid \leq 2)-N_{0}\left(r, 0 ; f^{\prime} \mid \leq 1\right) .
\end{aligned}
$$

So from (13) we get by (12) and Lemma 6 (ii) and (iv)

$$
\begin{aligned}
2 T(r, f) \leq & T(r, f)+T(r, g)-N_{0}(r) \\
& +N(r, a ; f \mid \leq 2)-N_{0}\left(r, 0 ; f^{\prime} \mid \leq 1\right)+S(r) \\
= & T(r, f)+N(r, a ; f \mid \leq 2)+S(r, f) \\
\leq & 2 T(r, f)+S(r, f),
\end{aligned}
$$

which is a contradiction.

Hence $f$ is a bilinear transformation of $g$. So any one of the possibilities of (ii)-(vii) of Lemma 5 will occur. We now examine each of these possibilities one by one.

Let $f+g \equiv 1$ Since $f, g$ share $(0,1),(1, m)$, it follows that 0 and 1 are Picard exceptional values (evP) of $f$ and so by the second fundamental theorem and Lemma 7 we get

$$
\begin{aligned}
T(r, f) & \leq \bar{N}(r, a ; f \mid \leq 2)+S(r, f) \\
& \leq N(r, a ; f \mid \leq 2)+S(r, f) \\
& \leq T(r, f)+S(r, f),
\end{aligned}
$$

a contradiction.

Let $(f-1)(g-1) \equiv 1$. Since $f, g$ share $(1, m),(\infty, k)$, it follows that 1 and $\infty$ are evP of $f$ and so as above we get $N(r, a ; f \mid \leq 2)=T(r, f)+$ $S(r, f)$, a contradiction.

If $f g \equiv 1$. Since $f, g$ share $(0,1),(\infty, k)$, it follows that 0 and $\infty$ are evP of $f$ and so $N(r, a ; f \mid \leq 2)=T(r, f)+S(r, f)$, a contradiction.

Let $f \equiv A g+1-A$, where $A(\neq 0,1)$ is a constant. Since $f, g$ share $(0,1)$, it follows that $0,1-A$ are evP of $f$ and so by the second fundamental theorem and Lemma 2 we get $T(r, f) \leq N(r, \infty ; f \mid \leq 1)+$ $S(r, f) \leq T(r, f)+S(r, f)$, a contradiction.

Let $f \equiv A g$, where $A(\neq 0,1)$ is a constant. Since $f, g$ share $(1, m)$, it follows that $1, A$ are evP of $f$ and so $N(r, \infty ; f \mid \leq 1)=T(r, f)+S(r, f)$, 
a contradiction.

Let $f(g+A-1) \equiv A g$, where $A(\neq 0,1)$ is a constant. Since $f, g$ share $(\infty, k)$, it follows that $\infty$ is an evP of $f$ and so of $g$.

If $A \neq a$, by the second fundamental theorem and Lemma 7 we get

$$
\begin{aligned}
T(r, f) & \leq \bar{N}(r, a ; f \mid \leq 2)+\bar{N}(r, a ; f)+S(r, f) \\
& \leq N(r, a ; f \mid \leq 2)+\bar{N}(r, \infty ; g)+S(r, f) \\
& =N(r, a ; f \mid \leq 2)+S(r, f) \\
& \leq T(r, f)+S(r, f),
\end{aligned}
$$

a contradiction.

Therefore $A=a$ and so $(f-a)(g+a-1) \equiv a(1-a)$. This proves the theorem.

Remark 1 If in Theorem 1 we remove the condition $N(r, \infty ; f \mid \leq 1) \neq$ $T(r, f)+S(r, f)$, in a like manner we can prove that one of the following possibilities occurs, which improves Theorem 4 [12]:

(i ) $(f-a)(g+a-1) \equiv a(1-a)$. This occurs only when $\infty$ is an evP of $f$. In this case $a, 1-a$ are evP of $f$ and $g$ respectively and $\infty$ is an evP of $g$.

(ii) $f+(a-1) g \equiv a$. This occurs only when 0 is an evP of $f$. In this case $a$ is an evP of $f$ and $0, a /(a-1)$ are evP of $g$.

(iii) $f \equiv a g$. This occurs only when 1 is an evP of $f$. In this case $a$ is an evP of $f$ and $1,1 / a$ are evP of $g$.

\section{Applications}

In this section we discuss two applications of Theorem 1.

Definition 5 ([3]) For $S \subset \mathbb{C} \cup\{\infty\}$ we define $E_{f}(S, k)$ as $E_{f}(S, k)=$ $\cup_{a \in S} E_{k}(a ; f)$, where $k$ is a nonnegative integer or infinity.

H.X. Yi [12] proved the following result.

Theorem $\mathbf{H}([12]) \quad$ Let $S_{1}=\left\{a_{1}, a_{2}\right\}$ and $S_{2}=\left\{b_{1}, b_{2}\right\}$ be two pairs of distinct elements with $a_{1}+a_{2}=b_{1}+b_{2}$ but $a_{1} a_{2} \neq b_{1} b_{2}$ and let $S_{3}=\{\infty\}$. If $E_{f}\left(S_{i}, \infty\right)=E_{g}\left(S_{i}, \infty\right)$ for $i=1,2,3$ and $\delta(c / 2 ; f)>0$ for $c=a_{1}+a_{2}$ then one of the following holds: ( i ) $f \equiv g$, (ii) $f+g \equiv a_{1}+a_{2}$, (iii) ( $f-$ $c / 2)(g-c / 2) \equiv \pm\left(a_{1}-a_{2}\right)^{2} / 4$, which occurs only for $\left(a_{1}-a_{2}\right)^{2}+\left(b_{1}-\right.$ $\left.b_{2}\right)^{2}=0$. 
H.X. Yi [12] considered the following example to establish the necessity of the condition $\delta(c / 2 ; f)>0$ for Theorem $H$.

Example 6 ([12]) Let $f=1-4 e^{z}, g=1-e^{-z}, a_{1}=-1, a_{2}=1, b_{1}=$ $-i \sqrt{3}, b_{2}=i \sqrt{3}, S_{1}=\left\{a_{1}, a_{2}\right\}, S_{2}=\left\{b_{1}, b_{2}\right\}$ and $S_{3}=\{\infty\}$. Then clearly $\left(f-a_{1}\right)\left(f-a_{2}\right)=-8 e^{2 z}\left(g-a_{1}\right)\left(g-a_{2}\right)$ and $\left(f-b_{1}\right)\left(f-b_{2}\right)=4 e^{z}(g-$ $\left.b_{1}\right)\left(g-b_{2}\right)$ so that $E_{f}\left(S_{i}, \infty\right)=E_{g}\left(S_{i}, \infty\right)$ for $i=1,2,3$. Also we see that $c=a_{1}+a_{2}=0, \delta(c / 2 ; f)=0$ and $f \not \equiv g, f+g \not \equiv a_{1}+a_{2},(f-c / 2)(g-$ $c / 2) \not \equiv \pm\left(a_{1}-a_{2}\right)^{2} / 4$.

In the following theorem we improve Theorem $H$ and show that the condition $\delta(c / 2 ; f)>0$ can further be relaxed.

Theorem 2 Let $S_{1}=\left\{a_{1}, a_{2}\right\}$ and $S_{2}=\left\{b_{1}, b_{2}\right\}$ be two pairs of distinct elements with $a_{1}+a_{2}=b_{1}+b_{2}$ but $a_{1} a_{2} \neq b_{1} b_{2}$ and let $S_{3}=\{\infty\}$. Suppose that $E_{f}\left(S_{1}, 1\right)=E_{g}\left(S_{1}, 1\right), E_{f}\left(S_{2}, m\right)=E_{g}\left(S_{2}, m\right), E_{f}\left(S_{3}, k\right)=$ $E_{g}\left(S_{3}, k\right)$ and $\delta_{1)}(c / 2 ; f)>0$, where $(m-1)(m k-1)>(1+m)^{2}$ and $c=$ $a_{1}+a_{2}$. Then the conclusion of Theorem $\mathrm{H}$ holds.

Proof. Let $A=\left(b_{1}-b_{2}\right)^{2} / 4-\left(a_{1}-a_{2}\right)^{2} / 4$ and

$$
F=\frac{1}{A}\left[\left(f-\frac{c}{2}\right)^{2}-\frac{\left(a_{1}-a_{2}\right)^{2}}{4}\right], G=\frac{1}{A}\left[\left(g-\frac{c}{2}\right)^{2}-\frac{\left(a_{1}-a_{2}\right)^{2}}{4}\right] .
$$

If $F \equiv G$ then clearly either $f \equiv g$ or $f+g \equiv a_{1}+a_{2}$. So we suppose that $F \not \equiv G$. Also let $\left(a_{1}-a_{2}\right)^{2}+\left(b_{1}-b_{2}\right)^{2}=0$ and $a=1 / 2$. Then we see that $A(F-a)=(f-c / 2)^{2}$ and so $N(r, \infty ; F \mid \leq 1) \equiv 0$ and $N(r, a ; F \mid \leq$ $2)=2 N(r, c / 2 ; f \mid \leq 1) \neq 2 T(r, f)+S(r, f)=T(r, F)+S(r, F)$. Since $F, G$ share $(0,1),(1, m),(\infty, k)$, by Theorem 1 we get $(F-a)(G+a-$ $1) \equiv a(1-a)$ and so $(f-c / 2)(g-c / 2) \equiv \pm\left(a_{1}-a_{2}\right)^{2} / 4$. This proves the theorem.

Remark 2 Example 6 shows that the condition $\delta_{1)}(c / 2 ; f)>0$ is essential.

In [5] following result is proved.

Theorem I $([5])$ Let $a$ and $b(\neq 0,1)$ be two finite complex numbers and $S_{1}=\left\{a+\alpha: \alpha^{n}+b=0\right\}, S_{2}=\left\{a+\beta: \beta^{n}+b=1\right\}, S_{3}=\{\infty\}$ where $n(\geq$ 3) be a positive integer. If $E_{f}\left(S_{1}, 1\right)=E_{g}\left(S_{1}, 1\right), E_{f}\left(S_{2}, \infty\right)=E_{g}\left(S_{2}, \infty\right)$, $E_{f}\left(S_{3}, \infty\right)=E_{g}\left(S_{3}, \infty\right)$ then one of the following holds: (i) $f-a \equiv t(g-$ $a)$, where $t^{n}=1$ and (ii) $(f-a)(g-a) \equiv s$, where $4 s^{n}=1$. 
In the next theorem we improve Theorem $I$.

Theorem 3 Theorem I holds if $E_{f}\left(S_{1}, 1\right)=E_{g}\left(S_{1}, 1\right), E_{f}\left(S_{2}, m\right)=$ $E_{g}\left(S_{2}, m\right)$ and $E_{f}\left(S_{3}, k\right)=E_{g}\left(S_{3}, k\right)$, where $(m-1)(m k-1)>(1+m)^{2}$.

We omit the proof as it can be done in the line of Theorem I using Theorem 1.

\section{References}

[1] Hayman W.K., Meromorphic Functions. The Clarendon Press, Oxford, 1964.

[2] Hua X. and Fang M., Meromorphic functions sharing four small functions. Indian J. Pure Appl. Math. vol. 28, 1997, pp. 797-811.

[3] Lahiri I., Weighted sharing and uniqueness of meromorphic functions. Nagoya Math. J. 161 (2001), pp. 193-206.

[4] Lahiri I., Weighted value sharing and uniqueness of meromorphic functions. Complex Variables Theory Appl. (3) 46 (2001), 241-253.

[5] Lahiri I., Weighted sharing of three values and uniqueness of meromorphic functions. Kodai Math. J. 24 (2001), 421-435.

[6 ] Lahiri I., On a result of Ozawa concerning uniqueness of meromorphic functions II. J. Math. Anal. Appl. (1) 283 (2003), 66-76.

[7] Lahiri I., Uniqueness of meromorphic functions and sharing of three values with some weight. New Zealand J. Math. (2) 32 (2003), 161-171.

[8] Lahiri I. and Sarkar A., On a uniqueness theorem of Tohge. Arch. Math. (Basel), (5) 84 (2005), 461-469.

[9] Ueda H., Unicity theorems for meromorphic or entire functions. Kodai Math. J. 3 (1980), 457-471.

[10] Ye S.Z., Uniqueness of meromorphic functions that share three values. Kodai Math. J. 15 (1992), 236-243.

[11] Yi H.X., Meromorphic functions that share three values. Chinese Ann. Math. Ser. A 9 (1988), 434-439.

[12] Yi H.X., Unicity theorems for meromorphic functions that share three values. Kodai Math. J. 18 (1995), 300-314. 